Article

\title{
Luminance Degradation Compensation Based on Multi-Stream Self-Attention to Address Thin Film Transistor-Organic Light Emitting Diode Burn-in
}

\author{
Seongchel Park@, Kwan-Ho Park and Joon-Hyuk Chang $(\mathbb{D}$ * \\ Department of Electronics and Computer Engineering, Hanyang University, Seoul 04763, Korea; \\ psc0902@hanyang.ac.kr (S. P.); rhksgh7370@naver.com (K.-H. P.) \\ * Correspondence: jchang@hanyang.ac.kr; Tel.: +82-2-2220-0355
}

\begin{abstract}
In this study, we propose a deep learning algorithm that directly compensates for luminance degradation owing to the deterioration of organic light emitting diode (OLED) devices to address the burn-in phenomenon of OLED displays. Conventional compensation circuits are encumbered by a high cost of development and manufacturing processes owing to their complexity. However, given that deep learning algorithms are typically mounted on a system on chip (SoC), the complexity of the circuit design is reduced, and the circuit can be reused by re-learning only the changed characteristics of the new pixel device. The proposed approach comprises deep feature generation and multi-stream self-attention, which decipher the importance of the variables, and the correlation between burn-in-related variables. It also utilizes a deep neural network that identifies the nonlinear relationship between the extracted features and luminance degradation. Thereafter, the luminance degradation is estimated from the burn-in-related variables, and the burn-in phenomenon can be addressed by compensating for the luminance degradation. The experimental results revealed that compensation was successfully achieved within an error range of $2.69 \%$, and demonstrate the potential of a new approach that can mitigate the burn-in phenomenon by directly compensating for pixel-level luminance deviation.
\end{abstract}

Keywords: thin film transistor (TFT); organic light emitting diode (OLED); compensation circuit; luminance degradation; artificial intelligence; deep neural network; convolutional neural networks

\section{Introduction}

Currently, two types of displays are widely used. The first is the liquid crystal display (LCD), which generates images by controlling the amount of light emitted by the backlight unit (BLU). The second is an organic light emitting diode (OLED), which generates an image by controlling the amount of current supplied to the OLED device. OLED displays have significant advantages such as high color reproduction ranges, low power consumption, high brightness, high contrast ratio and wide viewing angle [1-3]. However, despite their excellent performance, they are hindered by the burn-in phenomenon. This phenomenon is caused by the operating mechanism of OLED displays. Its panels are composed of thin film transistor (TFT)-OLED devices mounted on each pixel, and they function as follows. First, a voltage is applied to the TFT device. Second, the TFT device controls the amount of current supplied to the OLED element according to the applied voltage. Finally, the OLED device controls the brightness of the display by adjusting the luminance according to the supplied current. In this operation, the OLED device is exposed to high temperatures owing to its luminescence characteristics. If this situation persists, it leads to problems with the driving voltage deviation of the TFT device and luminance deviation of the OLED device. Eventually, as the usage time increases, the deterioration of the OLED device accelerates, and luminance degradation occurs [4,5]. Ultimately, the burn-in phenomenon is a major cause of the deterioration of the image and video quality over time 
[6-9]. Therefore, research on pixel compensation technology that effectively addresses the burn-in phenomenon of OLED displays is important to continuously provide high-quality images and videos to users.

Traditionally, the compensation technology of OLED displays is typically based on two types of compensation circuits. First, the internal compensation circuit controls the driving voltage of the TFT device with pixel circuits such as 5T1C or 4T0.5C to compensate for the luminance degradation. The internal circuit can compensate for the deviation in the driving voltage of the TFT device. However, when an internal compensation circuit is added, the structural design requirements of the TFT-OLED device become complex, and a highly sophisticated process is required [10]. Moreover, when this internal compensation circuit is utilized, it is difficult to miniaturize the pixels. Therefore, alternative circuit compensation methods are needed for the ultra-minimization of pixels that is necessary to develop high-resolution OLED displays. Second, the external compensation circuit is a mechanism that senses the characteristics of TFT elements inside the panel using sensing circuits from the outside. It then performs a compensation operation in the data voltage application section [11,12]. That is, this circuit is composed of various types of sensing devices. However, the compensation circuit is complicated because sensing and compensation operations are performed externally. An analog-to-digital converter (ADC) is required for sensing, in addition to memory for the storage of the sensing data. Thus, the cost of development is higher than that of the internal compensation circuit. Therefore, more effective technology is required to design and build a low-cost and high-performance compensation circuit.

In this study, we propose a deep learning algorithm that directly compensates for luminance degradation in real time by using a data-driven approach to address the disadvantages of internal and external compensation circuits. Deep learning is a machine learning paradigm that infers information and extracts features from the given data using multiple processing layers. The results of several studies have shown that deep learning facilitates improved performance compared to traditional approaches in a variety of applications that use sensor data [13-17]. In this study, the usage time, temperature, average brightness, data voltage deviation of the TFT device, current supplied from TFT to OLED, and luminance deviation of OLED are used as input data for the training of the proposed deep learning algorithm. In addition, the deviation between the initial luminance of the OLED device and the luminance degradation due to deterioration is used as the target data. As such, the target data is the luminance that is compensated, and this value is obtained by subtracting the degraded luminance value from the initial luminance. When a deep learning algorithm is trained using input data and target data composed of these variables, it performs as a novel circuit that directly estimates the luminance that requires compensation. Ultimately, it is possible to address the limitations of the existing internal and external compensation circuits, such as the complexity of circuit design, high cost, and difficulty of miniaturization. In addition, in the past, when TFT-OLED devices were changed, the compensation circuit had to be redesigned according to the new characteristics. However, the proposed deep learning algorithm can re-learn and reuse the characteristics of the new TFT-OLED device. We evaluated the performance of the model by calculating the deviation between the compensated luminance and the initial luminance in frames to evaluate the performance of the proposed method and to address the phenomenon problem, which is spread within an error range of $2.69 \%$.

The remainder of this paper is organized as follows. Section 2 describes the data simulator that models the deterioration mechanism of TFT-OLED devices and creates the main variables that related in burn-in phenomenon. Section 3 is an overview of data augmentation to improve the performance of deep learning algorithms. Section 4 outlines the deep learning based algorithm that directly compensates for the degraded OLED luminance. The experimental environment and results are described in Section 5. Finally, Section 6 summarizes the main conclusions of the study. 


\section{DATA SIMULATOR}

A data simulator is proposed to obtain the burn-in-related variables of TFT-OLED devices as similar to a typical environment. First, the proposed data simulator uses the specifications of LD420EUN-UHA1 as a Si:H transistor, which is a TFT model. Using the data simulator, it is possible to generate pixel-by-pixel data from 0 to $10,000 \mathrm{~h}$ in 100 -hour increments from the input video. In addition, it is possible to generate pixel data from OLED displays for low temperature, room temperature, and high temperature from $0^{\circ} \mathrm{C}$ to $60^{\circ} \mathrm{C}$. The data simulator is developed to create data similar to a typical environment by mixing white noise with the generated pixel data. The data generated in this study are used to train the deep learning model in Section 4; the deep learning model is used to examine the correlation between the variables related to the TFT-OLED device that fluctuates in real time and the luminance of the deteriorated OLED device.

Figure 1 shows a block diagram of the data simulator. The input video comprised of various content-specific videos has a total length of $120 \mathrm{~min}, 30 \mathrm{fps}$, and a pixel size of $400 \times 300$ pixels. The detailed configuration of the input videos of the data simulator is presented in Table 1. Table 2 lists the parameters used in this section.

Table 1: Specifications of input videos.

\begin{tabular}{ll}
\hline Contents & Specifications \\
\hline Content 1 (40 mins) & Documentary, Action, News, Sports \\
\hline Content 2 (40 mins) & Entertainment, Beauty, Animation, Car review \\
\hline Content 3 (40 mins) & Game, Cooking, Job introduction, Romance \\
\hline
\end{tabular}

The order of operation of the data simulator is as follows:

1. First, the data simulator outputs i) the average brightness per pixel $\left(\overline{B_{p}}\right)$ and ii) operation time $\left(t_{p}\right)$ from the input video. It also adds iii) a temperature condition $(T)$ between $0^{\circ} \mathrm{C}$ and $60^{\circ} \mathrm{C}$, which affects the deterioration of the TFT and OLED devices.

2. The previously obtained $\overline{B_{p}}, t_{p}, T$ variables are used to output iv) the operation time with weights per pixel $\left(t_{p}^{\prime}\right)$ and v) the degraded TFT data voltage $\left(V_{d, t_{p}^{\prime}=\gamma}\right)$ with the change of time and temperature. White noise is also mixed to create conditions similar to real-world environments.

3. $V_{d, t_{p}^{\prime}=\gamma}$ is used for each time and temperature to output vi) the degraded OLED current $\left(I_{d, t_{p}^{\prime}=\gamma}\right)$ of the TFT and to mix the white noise.

4. vii) Degraded OLED luminance $\left(L_{t_{p}^{\prime}=\gamma}\right)$ is observed using $I_{d, t_{p}^{\prime}=\gamma}$ for each time and temperature. viii) The initial OLED luminance $\left(L_{t_{p}}=0\right)$ is obtained directly from the input video. 


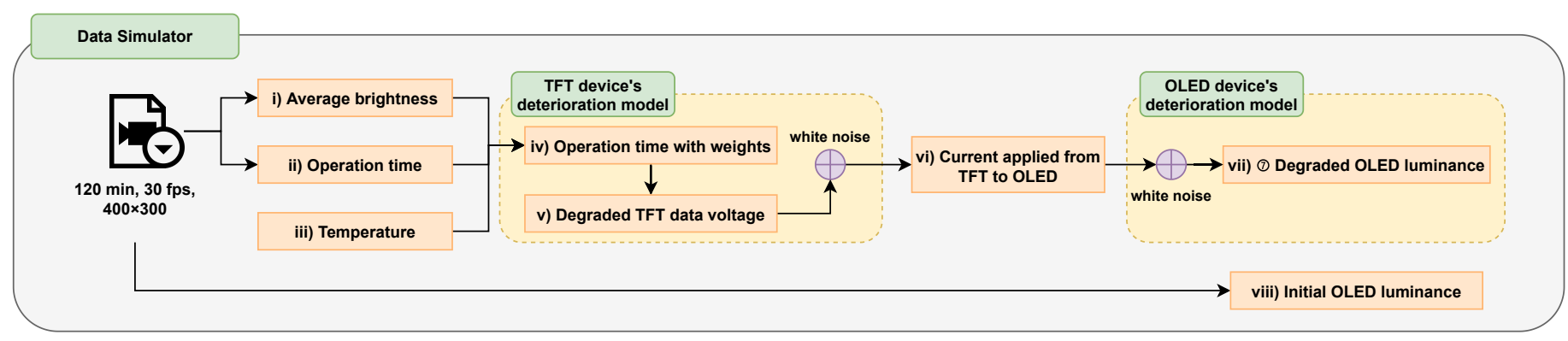

Figure 1. A block diagram of proposed Data Simulator.

Table 2: Nomenclature of paper.

\begin{tabular}{l|l|l|l}
\hline Symbol & Parameter & Symbol & Parameter \\
\hline$F_{N}$ & Data of input video & $N$ & Total frame of input video \\
$f$ & Frame & $P$ & Total pixel \\
$p$ & Pixel & $t$ & Time \\
$t_{p}$ & Operating time per pixel & $t_{p}^{\prime}$ & Weighted operating time \\
$B_{p}$ & Brightness of per pixel & $B_{p}$ & Average brightness per pixel \\
$\epsilon_{1}$ & Noise of threshold voltage & $\epsilon_{2}$ & Noise of mobility \\
$\alpha_{1}$ & Reduction factor of shifting value of threshold voltage & $\alpha_{2}$ & Reduction factor of threshold voltage \\
$\alpha_{3}$ & Reduction factor of mobility & $I_{\max }$ & Maximum input current of TFT \\
$L$ & Length of TFT channel & $W$ & Width of TFT channel \\
$V_{d}^{\prime}$ & Data voltage of TFT that consider noise & $V_{d, t_{p}=0}$ & Initial data voltage of TFT \\
$C_{o x}$ & Capacitor of TFT unit area & $\mu$ & Initial mobility of TFT \\
$V_{t h}^{\prime}$ & Threshold voltage of TFT that consider noise & $V_{\mathrm{DD}}$ & Drain voltage of TFT \\
$T_{\text {limit }}$ & Maximum temperature of TFT performance guarantee & $\triangle V_{\text {shift }}$ & Shifting value of threshold voltage \\
$V_{\mathrm{th}, t_{p}=0}$ & Initial threshold voltage of TFT & $w$ & Weight factor \\
$n$ & Gray level of TFT & $l$ & Total gray level range \\
$\alpha$ & Reduction rate of OLED voltage & $T$ & Temperature \\
$\beta$ & Transistor Parameter & $C_{i}$ & Gate capacitor \\
$W$ & Channel width & \\
\hline
\end{tabular}

Algorithm 1 shows the calculation process for the operation time and average brightness of the pixels for each frame from the video data input to the data simulator.

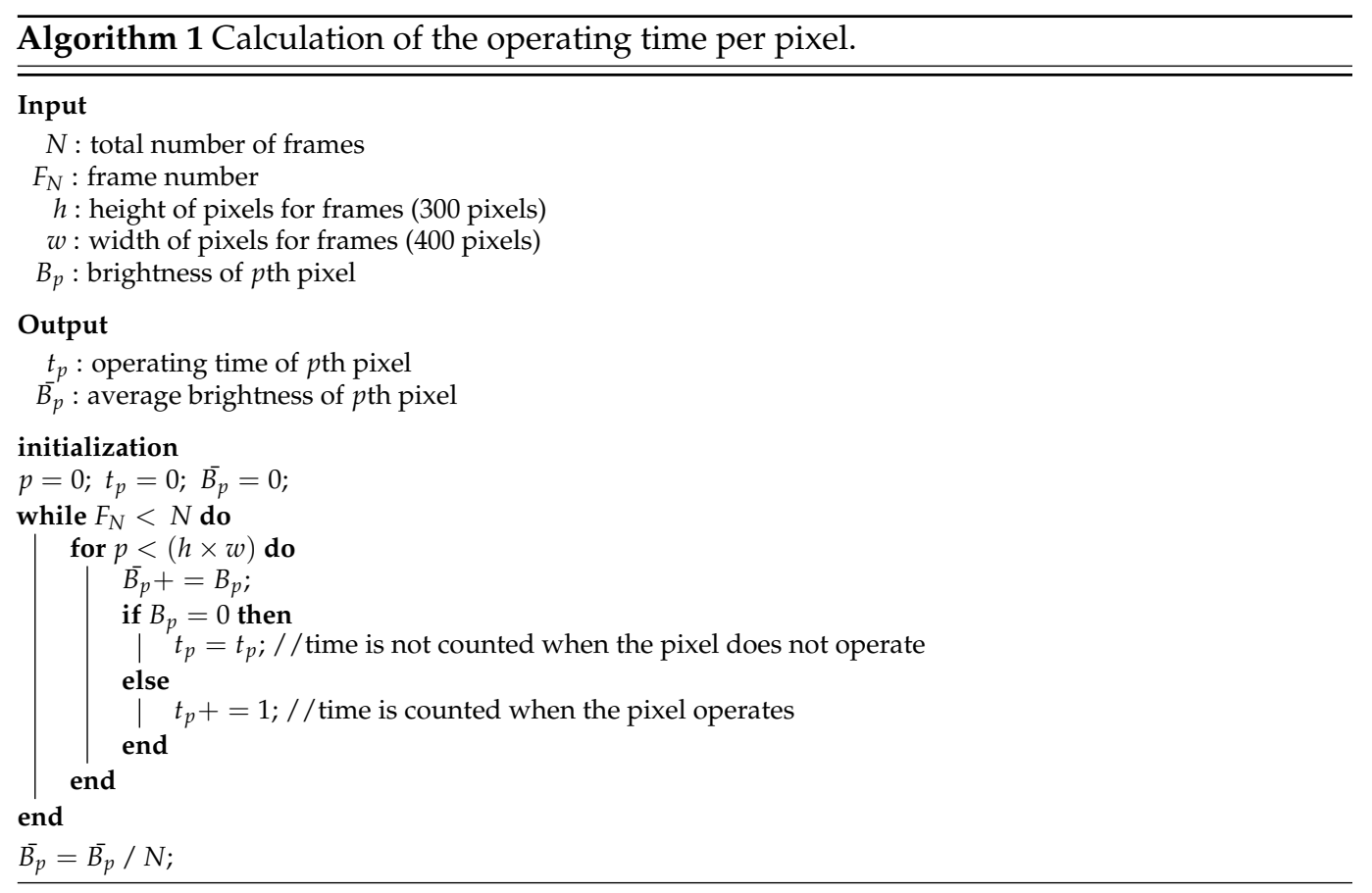


In particular, the average brightness and operation time of the input video obtained using this algorithm are used in equation (1) to obtain the weighted operation time $\left(t_{p}^{\prime}\right)$ required for the pixel to emit a specific brightness. Here, $\omega$ has a constant value of 0.8 and adjusts the weight to represent the average pixel brightness $\left(\bar{B}_{p}\right)$ during the operating time.

$$
t_{p}^{\prime} \triangleq t_{p}\left(1+w \overline{B_{p}}\right)
$$

We also generate white noise to represent the environmental noise that can occur in the OLED display using electronic circuits.

$$
\begin{gathered}
\epsilon_{1} \sim N\left(0, \frac{\left(\max \left(V_{\mathrm{th}}\right)+\min \left(V_{\mathrm{th}}\right)\right) / 2}{100}\right) \\
\epsilon_{2} \sim N\left(0, \frac{(\max (\mu)+\min (\mu)) / 2}{100}\right)
\end{gathered}
$$

Next, the threshold voltage shift value $\left(\Delta V_{\text {shift }}\right)$, threshold voltage $\left(V_{t h}\right)$, and electron mobility $(\mu)$ are calculated using the previously determined $t_{p}^{\prime}$ and $T$ value as follows.

$$
\begin{gathered}
\triangle V_{\text {shift }} \triangleq t_{p}^{\prime \alpha_{1}} \\
V_{\text {th }} \triangleq e^{\alpha_{2}\left(T-T_{\text {limit }}\right)}+\left|\Delta V_{\text {shift }}\right|+\epsilon_{1} \\
\mu \triangleq e^{-\alpha_{3} T}+\epsilon_{2}
\end{gathered}
$$

The data voltage $\left(V_{d, t_{p}^{\prime}=\gamma}\right)$ of the TFT is then obtained using $V_{t h}$ and $\mu$ [18]. Here, $V_{\mathrm{DD}}$ is the drain voltage of $4.9 \mathrm{~V}$ and additive noise, $\epsilon$.

$$
V_{d, t_{p}^{\prime}=\gamma} \triangleq V_{\mathrm{DD}}-\sqrt{\left(\frac{100}{100-\alpha}\right)\left(\frac{n}{l}\right) \frac{2 I_{\max }{ }^{\prime}}{\mu} C_{o x}\left(\frac{W}{L}\right)}-\left|V_{\mathrm{th}, t_{p}^{\prime}=\gamma}\right|+\epsilon
$$

Now, we use $V_{t h}$ and $V_{d, t_{p}^{\prime}=\gamma}$ to determine the current applied from the TFT to the $\operatorname{OLED}\left(I_{t_{p}^{\prime}=\gamma}\right)$ such that.

$$
I_{t_{p}^{\prime}=\gamma} \triangleq \frac{\beta}{2}\left(V_{\mathrm{DD}}-V_{d, t_{p}^{\prime}=\gamma}-\left|V_{\mathrm{th}}\right|\right), \beta=\mu C_{i} \frac{W}{L}
$$

where $I_{t_{p}^{\prime}=\gamma}$ is used to obtain the luminance value $\left(L_{t_{p}^{\prime}=\gamma}\right)$ at a specific time. The following is a mathematical model of the deterioration characteristics of the OLED device, where $m_{I}$ and $\eta_{I}$ have constant values [19].

$$
L_{t_{p}^{\prime}=\gamma} \triangleq L_{0} \exp \left\{-\left(\frac{t_{p}^{\prime}}{\eta_{I}\left(\frac{I_{0}}{I_{t_{p}^{\prime}=\gamma}^{\prime}}\right)^{\beta}}\right)^{m_{I}}\right\}
$$

When the video is served as the input to the data simulator, eight variables associated with the deterioration of the TFT-OLED device are created. Five of the eight variables are used as input data for the deep learning algorithm, and the luminance deviation obtained by subtracting the degraded OLED luminance value from the initial OLED luminance value is used as the target data. In addition, all pixels of the OLED are independently driven; therefore, the correlation between each pixel data generated by the data simulator is not considered. As such, 6,000 independent OLED burn-in data points are generated for each pixel between 0 to $10,000 \mathrm{~h}$ in units of $100 \mathrm{~h}$ and temperatures between $0^{\circ} \mathrm{C}$ to $60^{\circ} \mathrm{C}$ in units of $1^{\circ} \mathrm{C}$. The total burn-in datasets are generated 0.12 million $(400 \times 300)$ according to the number of pixels, and 720 million datasets $(100 \times 60 \times 400 \times 300)$ are generated according to time and temperatures for each color; $\mathrm{R}, \mathrm{G}$, and $\mathrm{B}$. 


\section{Data Augmentation}

In general, increasing the amount of data improves the performance of deep learning models [20]. Therefore, in this work, we generate additional data via data augmentation; subsequently, we conduct training using these data together with the existing data. The bootstrap method shown in Figure 2 is an approach for increasing the training data using random sampling. First, we calculate the mean and standard deviation of the data. These values are then used to construct a distribution plot for each characteristic, which then generates additional data along the distribution. This method is typically used, if the characteristics of the data must be generated as independent relationships. Finally, by applying the 720 million pixel data generated in the data simulator, 360 million training data with independent characteristics are additionally generated for each $R, G$, and $B$ color.

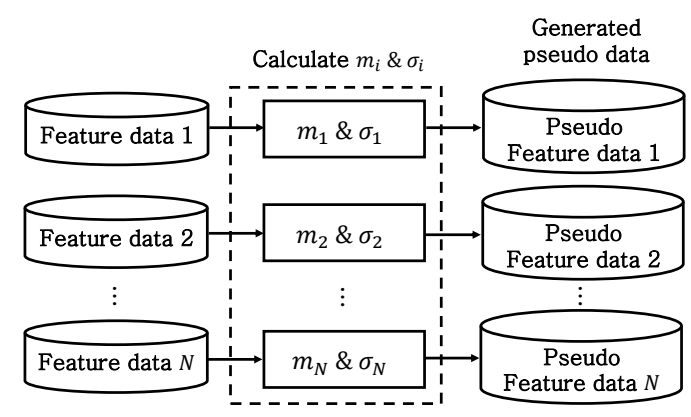

Figure 2. A block diagram of the bootstrap method.

\section{Deep Learning Model}

\subsection{Data Configuration}

The data used in the deep learning model consists of five features $\left(t_{p}^{\prime}, T, V_{d, t_{p}^{\prime}=\gamma^{\prime}}\right.$ $\left.I_{d, t_{p}^{\prime}=\gamma}, L_{t_{p}^{\prime}=\gamma}\right)$, consisting of vector forms with $(1,5)$ dimensions. The target data is one of the features, that is, the deviation luminance $\left(L_{t_{p}=0}-L_{t_{p}^{\prime}=\gamma}\right)$ that requires compensation. The total training data consists of 1.08 billion input data and target data pairs for each $\mathrm{R}$, $\mathrm{G}$, and B color. Figure 3 shows the structure of the entire deep learning model, which is trained to estimate the deviation luminance $\left(\hat{L}_{t_{p}^{\prime}=\gamma}\right)$, which requires compensation.

\subsection{Deep Feature Generation}

Feature generation is also known as feature construction, feature extraction or feature engineering. It is used to create new features from one or several features [21]. The implementation of this approach as a deep learning technique is called deep feature generation. Thus, in addition to the features generated using the data simulator, the features associated with OLED deterioration are also generated during the training process of the deep learning model to make them similar to the OLED burn-in data. In this study, we propose a deep feature generation algorithm composed of a 1D convolutional neural network (1D CNN), deep neural network (DNN), and rectified linear units (ReLU) [22], which are nonlinear functions, as shown in Figure 4. Using this algorithm, new features (embedding vectors) are also extracted using the existing input data with dimensions of $(1,5)$. First, 1D CNNs are available for 1D signal variables that cannot use 2D CNNs; the computational burden is lower than that of $2 \mathrm{D} \mathrm{CNNs}$, making them suitable for real-time processing and low-cost hardware implementations [23]. In addition, the DNN extracts information on the correlation between features by completely connecting the outputs of the 1D CNN. This DNN facilitates a nonlinear combination of input features and feature extraction is performed automatically. In the final output of this deep feature generation algorithm, 10 new features are generated with dimensions $(1,10)$. White noise is mixed to represent the noise in the OLED display circuit environment. Subsequently, one of the five 


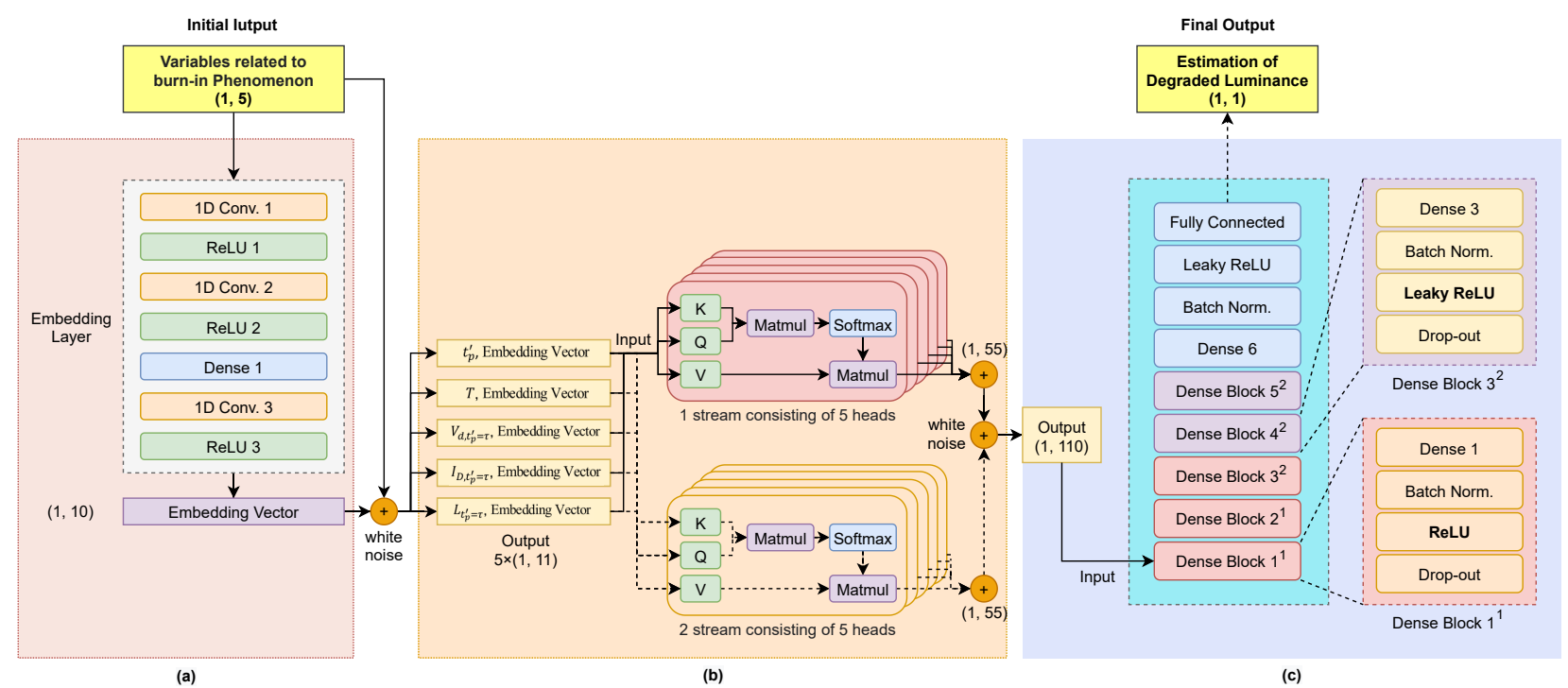

Figure 3. Overview of the whole model: (a) deep feature generation; (b) multi-stream self-attention; (c) deep neural network.

original features is selected and concatenated to the new features, resulting in a new form of data with a higher dimension $(1,11)$ than the original dimension $(1,5)$.

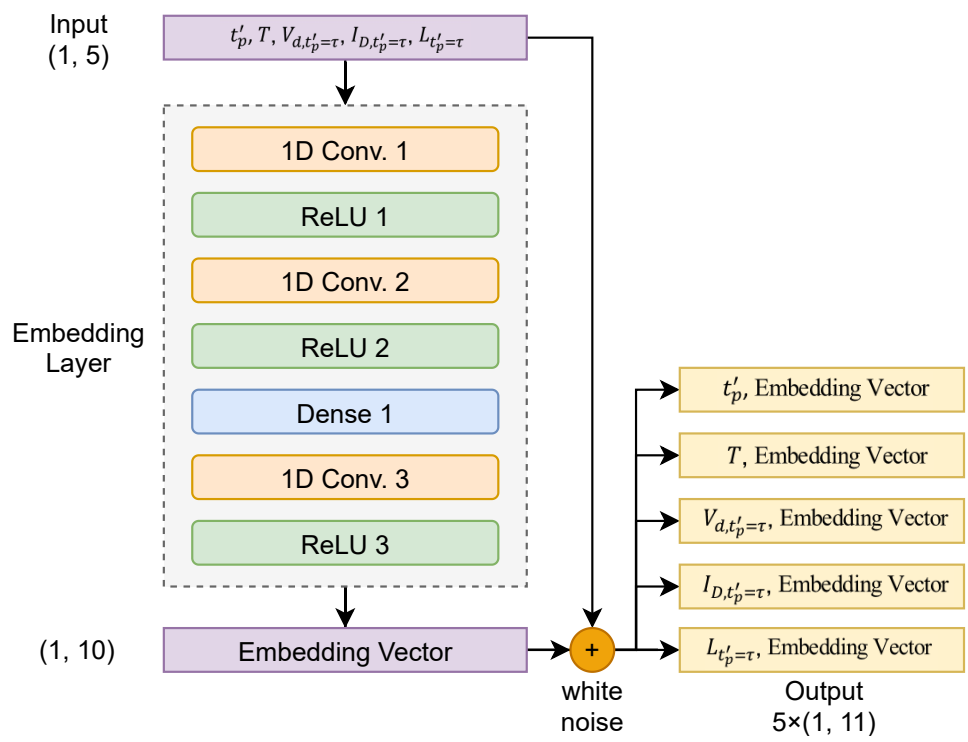

Figure 4. Overview of the deep feature generation model.

\subsection{Multi-stream Self-attention}

Multi-stream self-attention [24] consists of two multi-head self-attention layers [25] each of which consists of five self-attention layers, as shown in Figure 5. The operation process of this algorithm proceeds in the following order. First, multi-stream self-attention improves the performance of deep learning algorithms with ensemble-like effects. Second, the multi-head self-attention corresponding to each stream is trained by increasing the weight of the most important feature to effectively compensate for the degraded luminance among the input features. Similarly, less important features are trained such that the weight is reduced; that is, when five input data with dimensions $(1,11)$ are input to each head, an extraction process is performed that represents the importance of each feature by adjusting the weight value to focus on the most important of the 11 features. Third, given that the output of the multi-head self-attention maintains the dimension of the input data, the data 
with the $(1,55)$ dimension is output by concatenating five outputs of each head. Finally, multi-stream self-attention outputs data with dimensions $(1,110)$ as two outputs.

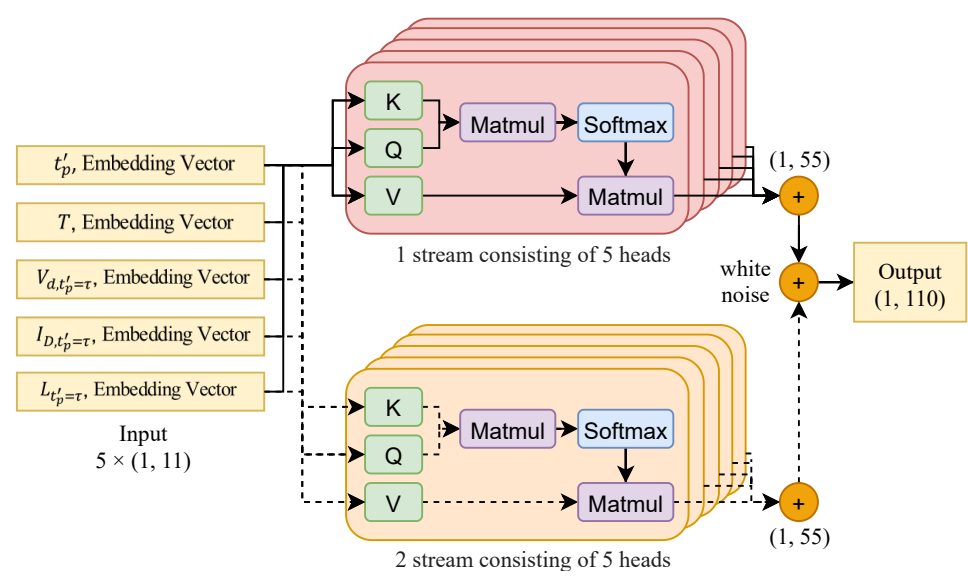

Figure 5. Overview of the multi-head self-attention model.

\subsection{DNN}

Recently, the DNN [26] has been successfully utilized in applications such as image processing, automatic speech recording, and natural language processing. As shown in Figure 6, the proposed algorithm consists of DenseBlock ${ }^{1}$, DenseBlock ${ }^{2}$, a single dense layer, and a fully connected layer. DenseBlock ${ }^{1}$ comprises of a single dense layer, batch normalization layer, ReLU, and dropout. DenseBlock ${ }^{2}$ is a nonlinear function of DenseBlock ${ }^{1}$, ReLU, replaced by Leaky-ReLU, which is proposed to solve the dying ReLU phenomenon. This DNN algorithm is trained to identify nonlinear relationships between input data and target data by recognizing specific patterns when data with dimensions $(1,110)$ are inputted. For the final output, we obtain the value of the dimension $(1,1)$ of luminance $\left(\hat{L}_{t_{p}^{\prime}=\gamma}\right)$ that requires compensation.

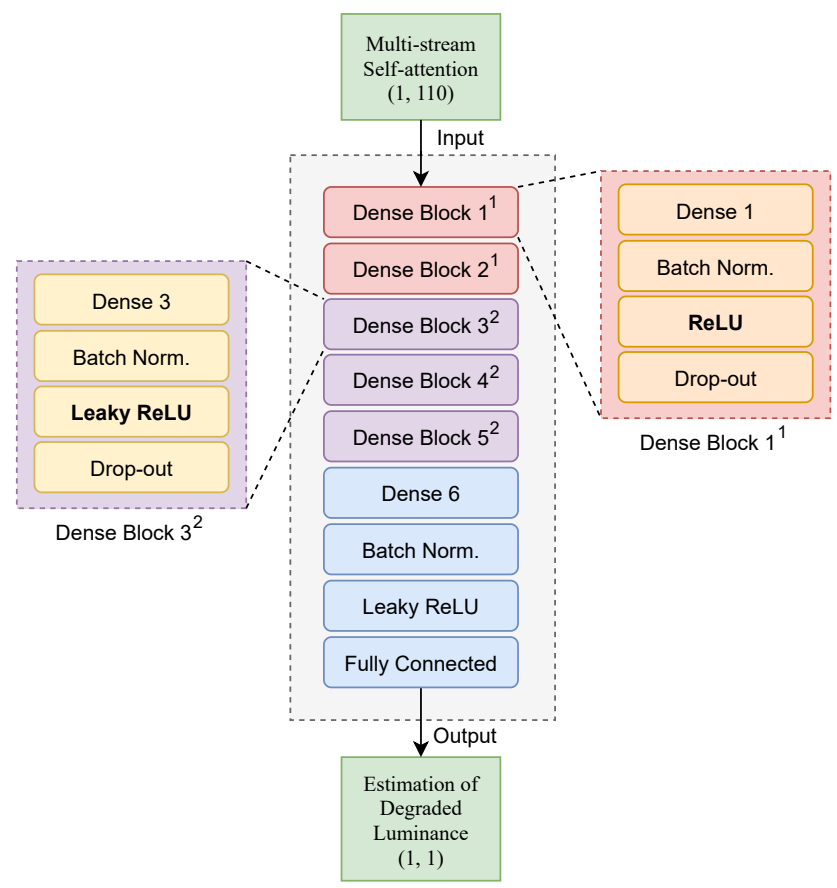

Figure 6. Overview of the Deep Neural Network model. 


\section{Experimental Environment and Result}

\subsection{Datasets}

In our experiments, we used the blue pixel data, which has the largest power consumption among burn-in data for R, G, and B, which have a much faster degradation rate compared to red and green [27]. Therefore, a deep learning based compensation algorithm was trained and evaluated using 1.08 billion datasets of blue pixel generated using data simulators and data augmentation. The compositions of the datasets, divided into training data and test data, are shown in Table 3.

Table 3: The composition of the datasets.

\begin{tabular}{c|c|c}
\hline Datasets & Train / Test & Total \\
\hline OLED pixel (Blue) & 9.72 billion / 1.08 billion & 10.8 billion \\
\hline
\end{tabular}

\subsection{Experimental Setup}

All experiments in this study were conducted using TensorFlow in Python library. Batch normalization [28] was applied to the DNN, and the Adam optimizer [29] with a learning rate of 0.001 was used for training a deep learning algorithm. In addition, the batch size used in the training process was 6,000, and all parts of the algorithm were jointly optimized with the mean absolute percentage error (MAPE) used as a loss function in the following. The algorithm was trained for 50 epochs; if the validation loss did not improve within three epochs, an early pause was applied. In addition, the accuracy of the algorithm was calculated using MAPE, as shown below.

$$
\begin{gathered}
\text { MAPE }=\frac{1}{N P} \sum_{f=1}^{N} \sum_{p=1}^{P}\left|\frac{L_{t_{p}=0}-\hat{L}_{t_{p}^{\prime}=\gamma}}{L_{t_{p}=0}}\right| \\
\text { Accuracy }=100\left(1-\frac{1}{N P} \sum_{f=1}^{N} \sum_{p=1}^{P}\left|\frac{L_{t_{p}=0}-\hat{L}_{t_{p}^{\prime}}=\gamma}{L_{t_{p}}=0}\right|\right)(\%)
\end{gathered}
$$

\subsection{Results Analysis}

As shown in Table 4, in the case of deep feature generation, experiments were conducted with three models; experiment 2 demonstrated the best accuracy at $92.76 \%$. As depicted in Table 5, based on the testing of the 1-stream self-attention and 2-stream self-attention algorithms. Experiment 2 showed an accuracy of $94.97 \%$. When using three or more streams, there was a tendency to overfit as the number of streams increased.

Table 5: Accuracy (in \%) comparison of the proposed models with Multi-stream Selfattention [24].

\begin{tabular}{c|c|c}
\hline $\begin{array}{c}\text { Experimental } \\
\text { Details }\end{array}$ & Experiment 1 & Experiment 2 \\
\cline { 2 - 3 } & 1-Stream Self-attention & 2-Stream Self-attention \\
\hline Accuracy & $92.31 \%$ & $\mathbf{9 4 . 9 7 \%}$ \\
\hline
\end{tabular}

In Table 6, experiments were conducted by changing the number of DNN layers in deep neural networks; experiment 2 demonstrated an accuracy of $95.04 \%$, which indicates that five layers are suitable. 
Table 4: Accuracy (in \%) comparison of the proposed models composed of different hyper-parameters with Deep Feature Generation (layers, kernel, filter size, and units).

\begin{tabular}{|c|c|c|c|c|c|c|}
\hline \multirow{3}{*}{$\begin{array}{c}\text { Experimental } \\
\text { Details }\end{array}$} & \multicolumn{2}{|c|}{ Experiment 1} & \multicolumn{2}{|c|}{ Experiment 2} & \multicolumn{2}{|c|}{ Experiment 3} \\
\hline & Layers & $\begin{array}{c}\text { Kernel } \\
\text { Filter Size } \\
\text { Units }\end{array}$ & Layers & $\begin{array}{c}\text { Kernel } \\
\text { Filter Size } \\
\text { Units }\end{array}$ & Layers & $\begin{array}{c}\text { Kernel, } \\
\text { Filter Size } \\
\text { Units }\end{array}$ \\
\hline & $\begin{array}{l}\text { 1D Conv } 1 \\
\text { 1D Conv } 2 \\
\text { Dense } 1\end{array}$ & $\begin{array}{c}1 \times 5 @ 32 \\
1 \times 32 @ 16 \\
16\end{array}$ & $\begin{array}{c}\text { 1D Conv } 1 \\
\text { 1D Conv } 2 \\
\text { Dense } 1 \\
\text { 1D Conv } 3\end{array}$ & $\begin{array}{c}1 \times 5 @ 32 \\
1 \times 32 @ 16 \\
16 \\
1 \times 16 @ 10\end{array}$ & $\begin{array}{l}\text { 1D Conv } 1 \\
\text { Dense } 1 \\
\text { 1D Conv } 2 \\
\text { Dense } 2 \\
\text { 1D Conv } 3\end{array}$ & $\begin{array}{c}1 \times 5 @ 32 \\
32 \\
1 \times 32 @ 16 \\
16 \\
1 \times 16 @ 10\end{array}$ \\
\hline Accuracy & \multicolumn{2}{|c|}{$91.44 \%$} & \multicolumn{2}{|c|}{$92.76 \%$} & \multicolumn{2}{|c|}{$92.15 \%$} \\
\hline
\end{tabular}

Table 6: Accuracy (in \%) comparison of proposed models with different numbers of Deep Neural Network's layers.

\begin{tabular}{c|cc|cc|cc}
\hline \multirow{2}{*}{$\begin{array}{c}\text { Experimental } \\
\text { Details }\end{array}$} & \multicolumn{2}{c|}{ Experiment 1 } & \multicolumn{2}{c|}{ Experiment 2 } & \multicolumn{2}{c}{ Experiment 3 } \\
\cline { 2 - 7 } & Layer number & Units & Layer number & Units & Layer number & Units \\
\cline { 2 - 7 } & Dense layer 1 & 64 & Dense layer 1 & 64 & Dense layer 1 & 64 \\
& Dense layer 2 & 64 & Dense layer 2 & 64 & Dense layer 2 & 64 \\
& Dense layer 3 & 64 & Dense layer 3 & 64 & Dense layer 3 & 64 \\
& Dense layer 4 & 64 & Dense layer 4 & 64 & Dense layer 4 & 64 \\
& & & Dense layer 5 & 64 & Dense layer 5 & 64 \\
& & & & & Dense layer 6 & 64 \\
\hline Accuracy & $92.44 \%$ & & \multicolumn{2}{c|}{$\mathbf{9 5 . 0 4 \%}$} & \multicolumn{2}{c}{$93.59 \%$} \\
\hline
\end{tabular}


Also, Table 7 shows the experimental results obtained by adjusting the number of units in each layer of the DNN algorithm. The DNN algorithm used five layers, as obtained from the experimental results in Table 6. As a result, experiment 4 with a bottleneck structure demonstrated an accuracy of $97.31 \%$.

Therefore, as shown in Tables 4, 5, 6, and 7, the final performance of the deep learning based compensation algorithm is the best accuracy of $97.31 \%$. Figure 7 compares the initial display image, the image in which luminance degradation occurred, and the image in which the degraded luminance was compensated.

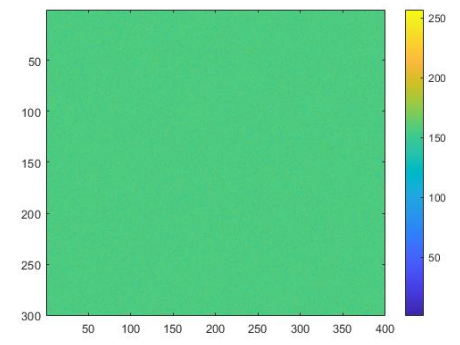

(a)

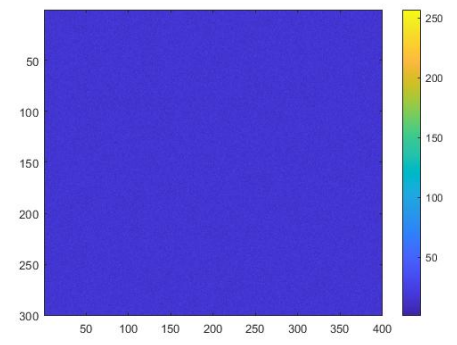

(b)

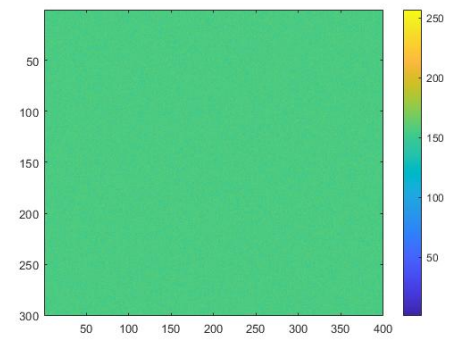

(c)

Figure 7. The image of OLED Display $(400 \times 300)$ according to the number of pixels: $(a)$ The initial display image; (b) The image in which luminance degradation occurred; (c) the image when degraded luminance was compensated.

\section{Conclusion}

In this study, we proposed the deep learning algorithm to address the burn-in phenomenon of OLED displays by using deep learning technology. This algorithm differs from physical circuit-based internal and external compensation circuits that compensate after sensing the luminance degradation value of TFT-OLEDs due to the deterioration of OLED devices. In particular, even if a new TFT-OLED device is developed, the significant advantage is that only the deep learning algorithm can be re-learned according to the parameters of the device and reused without the need to change the physical circuit. In addition, if OLED display is combined with cloud service, deep learning algorithm can be easily improved remotely. In the future, we will supplement the data simulator based on real data and augment the burn-in data to strengthen the deep learning based compensation algorithm.

Author Contributions: Conceptualization, Park, S., Park, K. and Chang, J. -H.; methodology, Park, S. and Park, K.; software, Park, S. and Park, K.; validation, Park, S.; writing, original draft preparation, Park, S.; writing, review and editing, Chang, J. -H.; supervision, Chang, J. -H.; project administration, Chang, J. -H.; funding acquisition, Chang, J. -H.

Conflicts of Interest: The authors declare no conflict of interest.

\section{References}

1. Kang, S.-. OLED power control algorithm using optimal mapping curve determination. J. Display Technol 2016, 12, pp. 1278-1282.

2. Jung, H.; Kim, Y.; Chen, C.; Kanicki, J. A-IGZO TFT based pixel circuits for AM-OLED displays. Proc. SID Tech. Dig 2012, pp. 1097-1100.

3. Wang, C.; Hu, Z.; He, X.; Liao, C.; Zhang, S. One gate diode-connected dual-gate a-IGZO TFT driven pixel circuit for active matrix organic light-emitting diode displays. IEEE Trans. Electron Devices 2016, 63, pp. 3800-3803.

4. In, H.-J.; Kwon, O.-K. External compensation of nonuniform Ph.electrical characteristics of thin-film transistors and degradation of OLED devices in AMOLED displays. IEEE Electron Device Lett. 2009, 30, pp. 377-379.

5. Lee, K.-Y.; Chao, Y.-P.; Chen, W.-D. A new compensation method for emission degradation in an AMOLED display via an external algorithm, new pixel circuit, and models of prior measurements. J. Display Techno. 2014, 10, pp. $189-197$.

6. Scholz, S.; Kondakov, D.; Lussem, B.; Leo, K. Degradation mechanisms and reactions in organic light-emitting devices. Chemical reviews 2015, 115, pp. 8449-5803. 
Table 7: Accuracy (in \%) comparison of the proposed models with different numbers of units of Deep Neural Network's layers.

\begin{tabular}{c|c|ccccc}
\hline \multirow{2}{*}{} & \multirow{2}{*}{ Layer number } & Experiment 1 & Experiment 2 & Experiment 3 & Experiment 4 & Experiment 5 \\
\cline { 3 - 7 } Experimental & & & Units & & \\
Details & & & 128 & 256 & 256 & 256 \\
& Dense layer 1 & 64 & 128 & 256 & 128 & 128 \\
& Dense layer 2 & 64 & 128 & 256 & 128 & 128 \\
& Dense layer 3 & 64 & 128 & 256 & 256 & 256 \\
& Dense layer 4 & 64 & 128 & 256 & 128 & 128 \\
& Dense layer 5 & 64 & 128 & 256 & 64 & 128 \\
\hline
\end{tabular}

7. Langlois, E.; Wang, D.; Shen, J. Degradation mechanisms in organic light emitting diodes. Synth. Metals 2000, 111, pp. $233-236$.

8. Schmidbauer, S.; Hohenleutner, A.; Konig, B. Chemical Degradation in Organic Light-Emitting Devices: Mechanisms and Implications for the Design of New Materials. Adv. Mater. 2013, 25, pp. 2114-2129.

9. Fery, C.; Racine, B.; Vaufrey, D.; Doyeux, H.; Cina, S. Physical mechanism responsible for the stretched exponential decay behavior of aging organic light-emitting diodes. Appl. Phys. Lett. 2005, 87, pp. 213502-213503.

10. Lee, K.-Y.; Hsu, Y.-P.; Chao, C.-P. A new 4T0.C AMOLED pixel circuit with reverse bias to alleviate OLED degradation. IEEE Electorn Device Lett. 2012, 33, pp. 1024-1026.

11. Lin, C.-L.; Chen, Y.-C. A novel LTPS-TFT pixel circuit compensating for TFT threshold-voltage shift and OLED degradation for AMOLED. IEEE Electron Device Lett. 2007, 28, pp. 129-131.

12. Lee, K.-Y.; Chao, C.-P. A new AMOLED pixel circuit with pulsed drive and reverse bias to alleviate OLED degradation. IEEE Electron Device Lett. 2012, 59, pp. 1123-1130.

13. Ravi, D.; Wong, C.; Lo, B.; Yang, G.-Z. One gate diode-connected dual-gate a-IGZO TFT driven pixel circuit for active matrix organic light-emitting diode displays. In Proc. 2016 IEEE 13th Int. Conf. Wearable Implantable Body Sensor Netw., pp. 71-76, Jun. 2016.

14. Zeng, M.; Nguyen, Le T.; Yu, Bo.; Mengshoel, Ole J.; Zhu, J.; Wu, P.; Zhang, J. Convolutional neural networks for human activity recognition using mobile sensors. In Proc. 2014 6th Int. Conf. Mobile Comput., Appl. Services, pp. 197-205, Nov. 2014.

15. Rav'1, D.; Wong, C.; Lo, B.; Yang, G. -Z. A Deep Learning Approach to on-Node Sensor Data Analytics for Mobile or Wearable Devices. IEEE Journal of Biomedical and Health Informatics 2017, 21.

16. Krizhevsky, A.; Sutskever, I.; Hinton, G. E. Imagenet classification with deep convolutional neural networks. Proc. Adv. Neural Inf. Process. Syst. 25 2012, pp. 1097-1105.

17. Psuj, G. Degradation mechanisms in organic light emitting diodes. Sensors 18 (2): 292 2018, https://doi.org/10.3390/s18010292

18. In, H.-J.; Kwon, O.-K. External Compensation of Non-uniform Electrical Characteristics of Thin-Film Transistors and Degradation of OLED Devices in AMOLED Displays. IEEE Electron Device Letters 2009, 30.

19. Zhang, J.; Li, W.; Cheng, G.; Chen, X.; Wu, H.; Shen, H. Life prediction of OLED for constant-stress accelerated degradation tests using luminance decaying model. Appl. Phys. Lett. 2014, 154, pp. 491-495.

20. Taylor, L.; Nitschke, G. Improving Deep Learning using Generic Data Augmentation. arXiv:1708.06020v1 2017.

21. Bosch, S. V. D. Automatic feature generation and selection in predictive analytics solutions. Master's thesis, Faculty of Science, Radboud University, vol. 3, no. 1, p. 3.1, 2017.

22. Agarap, F. Deep learning using rectified linear units (relu). arXiv preprint arXiv:1803.08375 2018.

23. Kiranyaz, S. 1-D Convolutional Neural Networks for Signal Processing Applications. In ICASSP 2019 - 2019 IEEE International Conference on Acoustics, Speech and Signal Processing (ICASSP), pp. 8360-8364, 2019.

24. Han, K. J.; Prieto, R. State-of-the-art speech recognitions using multi-stream self-attention with dilated 1D convolutions. arXiv:1910.00716v1 2019.

25. Vaswani, A.; Shazeer, N.; Parmar, N.; Uszkoreit, J.; Jones, L.; Gomez, A. N.; Kaiser, Ł.; Polosukhin, I. Attention is all you need. Advances in Neural Information Processing Systems 2017, pp. 5998-6008.

26. Heaton, J.; Goodfellow, I.; Bengio, Y.; Courville, A. Deep learning. Genet Program Evolvable Mach. 2018, https://doi.org/10.1007/ s10710-017-9314-z.

27. Yeh, C.-H. Visual-Attention-Based Pixel Dimming Technique for OLED Displays of Mobile Devices. IEEE Transactions on industrial electronics 2019, 66

28. Ioffe, S.; Szegedy, C. Batch normalization: Accelerating deep network training by reducing internal covariate shift. Available online: https://arxiv.org/pdf/1502.03167.pdf (accessed on 19 September 2019).

29. Kingma, D. P.; Ba, J. Adam: A method for stochastic optimization. Available online: https://arxiv.org/pdf/1412.6980.pdf (accessed on 19 September 2019). 